\title{
Pattern of Thyroid Cancer at King Abdulaziz University Hospital, Jeddah: A 10-Year Retrospective Study
}

\author{
Amani Alhozali, Aisha Al-Ghamdi, Jawaher Alahmadi \\ Department of Medicine, King Abdulaziz University Hospital, Jeddah, Saudi Arabia \\ Email: ahuzali@hotmail.com
}

Received 10 January 2016; accepted 14 March 2016; published 17 March 2016

Copyright (C) 2016 by authors and Scientific Research Publishing Inc.

This work is licensed under the Creative Commons Attribution International License (CC BY). http://creativecommons.org/licenses/by/4.0/

(c) (i) Open Access

\begin{abstract}
Background and objective: Thyroid cancer is the most common endocrine malignancy. This report aims to describe the pattern of thyroid cancer presentations at King Abdulaziz University Hospital, Jeddah. Methods: This was a retrospect chart review of all thyroid cancer cases diagnosed between 2001 and 2010 at King Abdulaziz University Hospital, Jeddah. We documented patients' demographic and clinical data, including age at diagnosis, tumor type and size, extrathyroidal extension, and metastasis. Results: A total of 114 thyroid cancer cases were diagnosed from 2001 through 2010. Females comprise the majority of cases (female to male ratio of $4: 1$ ). The mean ages of patients diagnosed with different thyroid cancers were: papillary thyroid cancer, 39.6 years; follicular thyroid cancer, 43.2 years; medullary thyroid cancer, 55.8 years; and anaplastic thyroid cancer, 46.0 years. Papillary thyroid cancer was diagnosed in 88 cases $(77 \%)$, follicular thyroid cancer in 19 cases $(17 \%)$, medullary thyroid cancer in 5 cases $(4 \%)$, and anaplastic thyroid cancer in 2 cases $(2 \%)$. Conclusion: Thyroid cancers are more common among females. The disease is diagnosed at a relatively young age among our patients (40 years). Papillary thyroid cancer is the most common type of thyroid cancer.
\end{abstract}

\section{Keywords}

Anaplastic Thyroid Cancer, Follicular Thyroid Cancer, Medullary Thyroid Cancer, Papillary Thyroid Cancer, Endocrine Malignancy

\section{Introduction}

Thyroid cancer is the most common endocrine malignancy and accounts for more than $90 \%$ of tumors of the

How to cite this paper: Alhozali, A., Al-Ghamdi, A. and Alahmad, J. (2016) Pattern of Thyroid Cancer at King Abdulaziz University Hospital, Jeddah: A 10-Year Retrospective Study. Open Journal of Endocrine and Metabolic Diseases, 6, 121-125. http://dx.doi.org/10.4236/ojemd.2016.63016 
endocrine system. In 2010, it ranked the third most prevalent cancer among Saudi adults, after breast and colorectal cancer [1]. It ranks the second among females after breast cancer and the twelfth among males in Saudi Arabia. An estimated 697 newly diagnosed thyroid cancer cases were recorded in Saudi Arabia in 2010 [1].

The incidence of thyroid cancer has increased substantially around the world. In the United States, the overall incidence rose from 1.3/100,000 for women and 4.6/100,000 for men in 1935 to 16.3/100,000 for women and $5.6 / 100,000$ for men in 2008 [2].

The objective of this study was to determine the prevalence of thyroid cancer among patients who were treated and followed up at King Abdulaziz University Hospital (KAUH), Jeddah, in the western region of Saudi Arabia. It also aims to describe the demographic and clinical characteristics of thyroid cancer patients.

\section{Methods}

This retrospective cohort study was conducted on patients who underwent thyroid surgery for nodular thyroid disease at KAUH, Jeddah, Saudi Arabia between January 2001 and December 2010. Patients older than 15 years with a proven histopathological diagnosis of thyroid cancer and who had received surgical treatment were included in this analysis. All cases of benign thyroid tumors and other malignancies (such as lymphomas and sarcomas) were excluded.

Patients' charts were reviewed using their medical record numbers after obtaining ethics committee approval. Medical records were reviewed for age at operation, gender, nationality, family history of thyroid cancer, and clinical presentation. We recorded histopathological findings and radioactive iodine (RAI) treatment (if administered).

Documented also were details of histopathology diagnosis, including the histopathological subtype, size of the tumor, presence of capsular and vascular invasion, extrathyroidal extension, and tumor surgical resection margin status. Lymph node involvement, distant metastasis, and mortality from thyroid malignancy were obtained.

Local recurrence was defined as histopathologically proven malignant thyroid tissue at the thyroid bed or cervical lymph nodes that required surgical resection or a second RAI dose.

\section{Statistical analysis:}

Data were analyzed using the Statistical Package for Social Science (SPSS Inc., IBM, US), version 16. Mean \pm standard deviation (SD) was calculated for quantitative data and proportions for categorical variables. The unpaired student's $t$-test was used to compare the means of continuous variables. Proportions were compared using the chi-square test. A P-value $<0.05$ was considered significant throughout the study.

\section{Results}

This study included 114 patients aged 40 years on average (range $15-87$ years; median, 38 years). The majority of cases were females $(n=91 ; 79.8 \%)$, representing a female to male ratio of $4: 1$. The mean age of the patients categorized by histopathological subtype was 39.6 years for papillary thyroid cancer (PTC), 43.2 years for follicular thyroid cancer (FTC), 55.8 years for medullary thyroid cancer (MTC) and 46.0 years for anaplastic thyroid cancer (ATC). Saudi patients accounted for $57 \%$ of the cases $(\mathrm{n}=65)$. Among non-Saudi patients, 38 were Asian (78\%) and the remainder African.

The majority of our patients presented with a right thyroid nodule $(\mathrm{n}=64 ; 52 \%)$, followed by a left thyroid nodule $(\mathrm{n}=42 ; 34 \%)$. The remaining cases were simple diffuse goiter and multinodular goiter (MNG), documented in $11 \%$ and $3 \%$ of the patients, respectively. Two patients presented with cervical lymphadenopathy, with normal thyroid gland.

A family history of thyroid cancer was documented in eight patients (7\%). A history of other malignancies was documented in two patients (1.6\%). Both patients were females, one of whom had breast cancer and the other colon cancer.

Total thyroidectomy and near total thyroidectomy were the most common surgical procedures performed in 106 patients (92\%). Eight patients $(7 \%)$ had either a right or left lobe thyroidectomy. Cervical lymph node dissection was performed in 18 patients $(15 \%)$.

Papillary thyroid carcinoma was diagnosed in 88 cases (77\%), followed by FTC, which was diagnosed in 19 cases (17\%) Table 1. Table 2 shows the histopathological subtypes of differentiated thyroid cancer. Most of the cases with PTC had tumors $<1 \mathrm{~cm}(\mathrm{n}=25) ; 17$ patients had tumor sizes $>4 \mathrm{~cm}$ (Table 2). Conversely, patients with FTC, in the majority of cases $(\mathrm{n}=9)$, had tumor size $>4 \mathrm{~cm}$; only one patient had a tumor $<1 \mathrm{~cm}$. In pa- 
Table 1. Frequency of histological types of thyroid carcinoma in the sample*.

\begin{tabular}{cccc}
\hline Cancer Types & Female & Male & Total \\
\hline Papillary thyroid carcinoma & 71 & 17 & $88(77.0)$ \\
Follicular thyroid carcinoma & 15 & 4 & $19(17.0)$ \\
Anaplastic thyroid carcinoma & 2 & 0 & $2(2.0)$ \\
Medullary thyroid carcinoma & 4 & 1 & $5(4.0)$ \\
\hline
\end{tabular}

*The data are presented as frequency and frequency (percent).

Table 2. Histological characteristic of thyroid carcinoma in the sample.

\begin{tabular}{cc}
\hline Histopathological Subtypes of DTC & Frequency (Percent) \\
\hline Non-follicular PTC & $64(60.0)$ \\
Follicular variant PTC & $21(20.0)$ \\
Hürthle cell & $8(7.0)$ \\
Insular variant & $5(5.0)$ \\
Invasive FTC & $3(3.0)$ \\
Oncocytic variant & $2(2.0)$ \\
Tall cell variant & $2(2.0)$ \\
Columnar cell variant & $1(0.9)$ \\
Oxyphilic cell variant & $1(0.9)$ \\
\hline
\end{tabular}

Abbreviations: DTC, differentiated thyroid carcinoma; FTC, follicular thyroid carcinoma; PTC, papillary thyroid carcinoma.

tients with MTC, tumor size ranged between 2.1 and $4 \mathrm{~cm}$ and greater, whereas patients with anaplastic thyroid carcinoma (ATC) had tumors $>4 \mathrm{~cm}$ in diameter.

Extrathyroidal extension to surrounding muscle and fat tissue was documented in 15 cases of PTC (16\%), 1 case of FTC (4.5\%), and all cases of ATC (100\%). Lymph node metastasis was confirmed in 25 cases of PTC (27\%), 1 case of FTC (4.5\%), 2 cases of MTC (40\%), and all cases of ATC (100\%). Local recurrence at the thyroid bed or cervical lymph node was documented in 10 cases of PTC (11\%), 1 case of FTC (4.5\%), and none of the other tumor variants (Table 3 ).

Distant metastasis was documented in 14 cases of PTC (15\%). Of these, seven patients had liver metastasis, four had lung metastasis, two had multiple-organ metastasis (liver, brain, and lung), and one had mediastinal metastasis. On the contrary, in patients with FTC, only two cases (9\%) had distant metastasis (one case to the liver and the other to the lung). Distant metastasis was documented in two patients with MTC (40\%), whereas neither of the two patients with ATC had metastatic disease.

Two patients with PTC died, which yielded a mortality rate of $1.6 \%$. One patient died from uncontrolled carotid bleeding due to thyroid infiltration by malignant cells. The other died from massive hemoptysis and respiratory distress caused by metastatic invasion of the trachea.

Further analysis demonstrated a mild significant association between PTC and younger age $(\mathrm{P}=0.09)$. In addition, patients with PTC were more likely to have distant metastasis $(\mathrm{P}=0.006)$.

\section{Discussion}

In our study, $80 \%$ of patients with thyroid cancer were female. The median age of patients in our study was 38 years, similar to that of patients documented in the Saudi cancer registry (47 years among males and 38 years among females) for the year 2010 [1]. In a study conducted at King Faisal Specialist Hospital and Research Center (KFSH \& RC), from 2000 to 2010, thyroid cancer patients comprised $76.3 \%$ females and $23.7 \%$ males [3]. 
Table 3. Staging of thyroid carcinoma in the sample.

\begin{tabular}{ccccc}
\hline Variables & PTC & FTC & ATC & MTC \\
\hline Tumor size & No & No & No & 0 \\
$<1 \mathrm{~cm}$ & 25 & 1 & 0 & 0 \\
$1.1-2 \mathrm{~cm}$ & 24 & 5 & 0 & 2 \\
$2.1-4 \mathrm{~cm}$ & 22 & 4 & 0 & 3 \\
$>4 \mathrm{~cm}$ & 17 & 9 & 2 & 2 \\
Extra thyroidal extension & 15 & 1 & 2 & 0 \\
Lymph node metastasis & 25 & 1 & 0 & 2 \\
Local recurrence & 10 & 1 & 0 & 0 \\
Distant metastasis & 14 & 2 & 0 & 2
\end{tabular}

Abbreviations: ATC, anaplastic thyroid carcinoma; DTC, differentiated thyroid carcinoma; FTC, follicular thyroid carcinoma; MTC, medullary thyroid carcinoma; PTC, papillary thyroid carcinoma.

In our study, most of the patients presented with right thyroid nodules, followed by left thyroid nodule. Papillary thyroid cancer comprised about $85 \%$ of cases compared with about $12 \%$ of FTC cases in a study conducted in the United States [4]. In our study, PTC was the most frequently diagnosed thyroid cancer and accounted for $77 \%$ of all thyroid cancer cases at KAUH. This is similar to national and international figures. A previous study conducted at KAUH in 2002 showed that PTC was the most common type of thyroid cancer (82\%). Another study from the central region of Saudi Arabia reported PTC in 89\% of thyroid cancer cases [5] [6]. In the eastern province, some investigators reported papillary thyroid cancer in $74 \%$ of their cohort [7]. Another study at KFSH \& RC revealed that papillary adenocarcinomas accounted for $72 \%$ of all thyroid cancers [3], whereas other investigators at the same institution found that $83.3 \%$ of their patients had PTC [8].

Follicular thyroid cancer accounted for $17 \%$ of the sample in our study. This frequency is higher than that reported in other studies conducted in Saudi Arabia. Alamoudi et al. [8] found that FTC accounted for 3.7\% of all thyroid cancers diagnosed between January 2000 and September 2006 at KFSH \& RC.

In the current study, MTC accounted for $4 \%$ of thyroid cancers, similar to the rate reported in studies conducted abroad [4]. In a study at KFSH \& RC, Jeddah, Saudi Arabia, 4.6\% of thyroid cancers were MTC [8].

This study has all the limitations inherent to retrospective chart reviews. In addition, data were missing in the electronic health records because some patients were referral cases from other hospitals or cities. As a result, we had to collect data from the hospital archives.

\section{Conclusion}

Overall, thyroid cancer is more common in females, with a female to male ratio of $4: 1$. Patients in our setting tend to present with thyroid cancer at a young age (40 years on average). Most patients present with neck lumps and PTC is the most common histopathological variant.

\section{References}

[1] Kingdom of Saudi Arabia, Ministry of Health and Saudi Cancer Registry (2010) Cancer Incidence Report.

[2] National Cancer Institute (2011) SEER Stat Fact Sheets: Thyroid Cancer. http://seer.cancer.gov/statfacts/html/thyro.html

[3] Hussain, F., Iqbal, S., Mehmood, A., Bazarbashi, S., El-Hassan, T. and Chaudhri, N. (2013) Incidence of Thyroid Cancer in the Kingdom of Saudi Arabia, 2000-2010. Hematology/Oncology and Stem Cell Therapy, 6, 58-64. http://dx.doi.org/10.1016/j.hemonc.2013.05.004

[4] Hundahl, S.A., Cady, B., Cunningham, M.P., Mazzaferri, E., McKee, R.F., Rosai, J., Shah, J.P., Fremgen, A.M., Ste- 
wart, A.K. and Hölzer, S. (2000) Initial Results from a Prospective Cohort Study of 5583 Cases of Thyroid Carcinoma Treated in the United States during 1996. U.S. and German Thyroid Cancer Study Group. An American College of Surgeons Commission on Cancer Patient Care Evaluation Study. Cancer, 89, 202-217. http://dx.doi.org/10.1002/1097-0142(20000701)89:1<202::AID-CNCR27>3.0.CO;2-A

[5] Qari, F.A. (2004) Pattern of Thyroid Malignancy at a University Hospital in Western Saudi Arabia. Saudi Medical Journal, 25, 447-451.

[6] Al-Sobhi, S.S. (2002) The Current Pattern of Thyroid Surgery in Saudi Arabia and How to Improve It. Annals of Saudi Medicine, 22, 3-4.

[7] Al-Amri, A.M. (2012) Pattern of Thyroid Cancer in the Eastern Province of Saudi Arabia: University Hospital Experience. Journal of Cancer Therapy, 3, 187-191. http://dx.doi.org/10.4236/jct.2012.33027

[8] Alamoudi, O., Hamour, O.A., Mudawi, I., Khayyat, A., Batwail, N. and Elhadd, T.A. (2011) Consensus Based Management of Differentiated Thyroid Cancer in Tertiary Care Set-Up. International Journal of Surgery, 9, 96-100. http://dx.doi.org/10.1016/j.ijsu.2010.10.005 